\title{
PROPRIEDADES FÍSICAS E VELOCIDADE MÍNIMA DE FLUIDIZAÇÃO DE PARTÍCULAS SEMENTES EMPREGADAS EM PROCESSOS DE AGLOMERAÇÃO DE PRODUTOS FARMACÊUTICOS E ALIMENTÍCIOS
}

\author{
L. BENELLI ${ }^{1 *}$, W. P. OLIVEIRA ${ }^{1}$ \\ ${ }^{1}$ Universidade de São Paulo, Faculdade de Ciências Farmacêuticas de Ribeirão Preto, \\ Departamento de Ciências Farmacêuticas \\ *e-mail: lucimarabenelli@gmail.com
}

\begin{abstract}
RESUMO
Neste trabalho foram determinadas propriedades físicas e características fluidodinâmicas de leitos fluidizados, constituídos de partículas sementes adequadas para utilização em processos de aglomeração/encapsulação nas áreas farmacêuticas e de alimentos. As partículas estudadas foram: açúcar cristal, pellets de açúcar, celulose microcristalina, pellets de celulose microcristalina, arroz e farinha de mandioca, sendo caracterizadas quanto às propriedades físico-químicas, granulometria, polidispersidade, fator de forma, características de fluidez, e perfil fluidodinâmico no sistema. Os resultados demonstraram que as partículas analisadas apresentam características físicoquímicas adequadas e bom comportamento fluidodinâmico, sendo ideais para serem empregadas como partículas sementes em processos de aglomeração e/ou revestimento em leito fluidizado.
\end{abstract}

\section{INTRODUÇÃO}

Processos em leitos fluidizados são amplamente utilizados em operações de mistura, secagem, revestimento, granulação, obtenção de sistemas de liberação controlada, etc., em vários setores industriais, incluindo o farmacêutico, de alimentos, químico e agrícola (Niamnuy e Devahastin, 2005; Murthy e Joshi, 2007; Mörl, Heinrich e Peglow, 2007; Dixit e Puthli, 2009; Yoshinobu e Hideki, 2013).

Nestes processos, a fluidização promove a ocorrência simultânea da secagem, revestimento e aglomeração de partículas em um único passo, reduzindo custos operacionais e tempo de processo, gerando produtos com características físico-químicas adequadas, como melhor fluidez, compressibilidade e uniformidade; mascaramento de sabores e odores desagradáveis; proteção contra deterioração por exposição ao oxigênio, umidade, luz e agentes incompatíveis; produtos com propriedades de liberação controlada, etc.. (Parikh e Mogavero, 2005; Saleh e Guigon, 2007; Benelli et al., 2015).

A aglomeração e/ou revestimento em leito fluidizado é um processo complexo que consiste na atomização de uma formulação de alimentação em um leito de partículas sementes sob fluidização devido à passagem de um fluxo de ar ascendente à determinada temperatura, resultando em grânulos aglomerados/revestidos.

Este processo sofre a influência de muitas variáveis inter-relacionadas como as propriedades físico-químicas das partículas sementes (densidade, tamanho, distribuição, forma, características da superfície e porosidade), da composição de alimentação 
(viscosidade, densidade, tensão superficial, ângulo de contato), da configuração do leito (tipo e posição do atomizador, design da placa distribuidora de ar, altura e largura da coluna do leito, etc.) e das variáveis operacionais (temperatura, umidade e vazão do ar de fluidização, vazão da formulação de atomização, pressão e vazão do ar de atomização, etc.).

O regime de fluidização no leito depende do tamanho inicial e da densidade das partículas sementes, que foram classificadas por Geldart (1973) em quatro grupos de acordo com as propriedades de fluidização: grupo C (partículas coesivas, muito finas, menores que $50 \mu \mathrm{m}$, e/ou baixa densidade aparente, de difícil fluidização), grupo A (partículas aeráveis, com tamanho entre 50 e $200 \mu \mathrm{m}$, e densidade entre 700 a $1400 \mathrm{~kg} / \mathrm{m}^{3}$, que fluidizam homogeneamente com baixas velocidades de gás, apresentando fluidização borbulhante), grupo B (partículas tipo areia tamanho entre 40 e $500 \mu \mathrm{m}$, com densidade entre 1400 a $4000 \mathrm{~kg} / \mathrm{m}^{3}$, fluidizam em regime pistonado de forma não homogênea) e grupo D (partículas jorráveis, grandes e/ou muito densas, que fluidizam em velocidades de gás muito altas, apresentando tendência a jorrar ao invés de fluidizar) (Geldart, 1973).

Portanto, o estudo das propriedades das partículas sementes e de sua fluidodinâmica é de fundamental importância para $o$ entendimento do fenômeno de transferência de calor e massa, do comportamento das partículas no interior do leito e do controle adequado do processo em leito fluidizado, auxiliando na seleção das condições operacionais mais adequadas para obtenção do produto final com as características desejadas.

Neste trabalho, foi realizado o estudo das características fluidodinâmicas em leito fluidizado e das propriedades físico-químicas de diferentes materiais particulados inertes de origem natural com potencial para serem utilizados como partículas sementes em processos, tanto na área farmacêutica como de alimentos. As partículas estudadas foram: açúcar cristal, pellets de açúcar, celulose microcristalina, pellets de celulose microcristalina, arroz e farinha de mandioca. Estas partículas apresentam vantagens por serem quimicamente inertes, apresentarem baixa toxicidade, baixo custo, serem biodegradáveis e biocompatíveis e estarem largamente disponíveis no mercado.

As partículas foram caracterizadas quanto à granulometria, polidispersidade, fator de forma e propriedades de fluidez, sendo, também, determinado os perfis fluidodinâmicos em leito fluidizado.

\section{MATERIAL E MÉTODOS}

\subsection{Material}

Partículas sementes: Açúcar cristal (faixa granulométrica de 300 a $500 \mu \mathrm{m}$ ); pellets de açúcar $\left(\right.$ Suglets ${ }^{\circledR}$, Colorcon do Brasil Ltda, PF006 - faixa granulométrica de 500 a $600 \mu \mathrm{m}$ e PF001 - faixa granulométrica de 255 a $355 \mu \mathrm{m}$ ); celulose microcristalina (Colorcon do Brasil Ltda - faixa granulométrica de 300 a $425 \mu \mathrm{m}$ ); pellets de celulose microcristalina $\left(\right.$ Celphere ${ }^{\circledR}$, Asahi Kasei Chemicals, Japan, CP 507 - faixa granulométrica de 500 a $710 \mu \mathrm{m}$ e CP 305 faixa granulométrica de 300 a $500 \mu \mathrm{m}$ ); farinha de mandioca seca, tipo I, acidez baixa (Marca Yoki, faixa granulométrica de 300 a $500 \mu \mathrm{m})$; e arroz tipo I beneficiado, polido, longo fino (Serra Azul), inicialmente moído em moinho de facas, modelo MA-680, Marconi (Piracicaba, SP, Brasil) até atingir a faixa granulométrica de 300 a $500 \mu \mathrm{m}$. As partículas foram classificadas por 
peneiramento em agitador de peneiras Bertel (São Paulo, Brasil).

Leito fluidizado: $\mathrm{O}$ equipamento experimental utilizado no desenvolvimento deste trabalho é constituído por uma coluna de fluidização de vidro, com diâmetro interno de $150 \mathrm{~mm}$ e altura de $5000 \mathrm{~mm}$. A coluna de vidro é acoplada a uma base de aço inoxidável, semi-cônica, com abertura inferior de $110 \mathrm{~mm}$ e altura de $100 \mathrm{~mm}$. Uma expansão do sistema foi conectada na parte superior do leito para reduzir a velocidade do gás, diminuindo a elutriação de partículas finas. Uma placa perfurada distribuidora do ar de fluidização foi colocada na parte inferior da base semi-cônica. Abaixo desta placa foi colocada uma peneira fina de aço (\# 200) para evitar a passagem de finos.

\subsection{Métodos}

Caracterização das partículas inertes: As partículas sementes foram caracterizadas quanto ao diâmetro médio, à distribuição granulométrica, índice de polidispersidade (SPAN), fator de forma e propriedades de fluidez. O diâmetro médio e a distribuição de tamanhos das partículas foram determinados por análise de imagem, utilizando-se uma máquina fotográfica digital Canon Power Shot SX50 HS (12,1 Megapixel), e analisados por um Sistema de Análise de Imagens (Image Pro-plus ${ }^{\circledR}$ 7.0). $\mathrm{O}$ índice de polidispersidade das partículas ou SPAN foi determinado de acordo com a literatura (Zili, Souad e Fessi, 2005). O fator de forma das partículas, $(\Phi)$, foi determinado através da medição dos valores médios de três dimensões características das partículas obtidas através do Sistema de Análises de Imagens (Image-Pro Plus ${ }^{\circledR}$ 7.0), sendo o resultado da razão entre o diâmetro menor e o diâmetro maior das partículas. As propriedades de fluidez das partículas foram avaliadas através do fator de Hausner $(\mathrm{FH})$ e o índice de Carr (IC) (Aulton, 2002), obtidos através da densidade bruta e de compactação das partículas. A densidade de compactação é obtida pela razão entre a massa e o volume ocupado pelas partículas após 1250 batidas a uma altura de $14 \mathrm{~mm}$, utilizando-se um medidor de densidade compactada de pó CALEVA TDT 1-23 (Alemanha).

Caracterização fluidodinâmica do sistema: A caracterização fluidodinâmica do leito fluidizado contendo as partículas sementes foi realizada através da determinação da velocidade mínima de fluidização (Umf) e queda de pressão máxima no leito $(\Delta \mathrm{Pmf})$. Para determinação destes parâmetros foram construídos gráficos da variação de pressão no leito em função da vazão do gás de fluidização. Com a massa de partículas no leito, a vazão do ar injetado foi variando de forma crescente, medindo-se a queda de pressão através de um sistema de aquisição de dados constituído por termopares, manômetro, micromanômetro e transdutores de pressão diferenciais nas faixas de $-2,50$ a 2,50 in $\mathrm{H}_{2} \mathrm{O}$ (modelo Omega 164PC01D37) e de 0 a 10 in $\mathrm{H}_{2} \mathrm{O}$ (modelo Omega 163PC01D75), conectados a uma placa de aquisição de dados PCL 711-S (Advantech) instalada em um microcomputador contendo o software Labtech (versão 3.2). O sistema foi configurado para coletar (de forma independente) quatro pontos por segundo. Atingindo-se a fluidização iniciou-se o procedimento inverso, diminuindo-se gradualmente a vazão do ar e medindo-se a queda de pressão.

\section{RESULTADOS E DISCUSSÕES}

Num processo em leito fluidizado, as partículas sementes constituem uma variável dependente que apresenta papel fundamental no desempenho do processo e nas propriedades do produto final. As características físico-químicas das partículas, como distribuição de tamanho e densidade 
exercem um papel fundamental na fluidodinâmica do sistema. Neste trabalho, partículas de diferentes estruturas e tamanhos foram analisadas quanto às suas características físico-químicas e comportamento fluidodinâmico em leito fluidizado. Na Tabela 1 estão apresentadas as características físico-químicas relacionadas com a distribuição de tamanho e forma das partículas e na Tabela 2 as densidades e propriedades de fluidez.
Os resultados mostram que as partículas obtidas por esferonização, como os pellets de açúcar e de celulose, apresentam maiores fator de forma, sendo que valor igual a 1 é representativo de uma esfera perfeita. Por outro lado, as partículas que têm formas mais irregulares como a farinha de mandioca, a celulose microcristalina e o arroz, apresentaram menor fator de forma e maior polidispersidade.

Tabela 1 - Caracterização físico-química das partículas inertes relacionadas à distribuição granulomética e fator de forma.

\section{Propriedades}

\begin{tabular}{ccccccc}
\hline Partículas & $\begin{array}{c}\mathrm{d}_{\mathrm{m}} \\
(\mu \mathrm{m})\end{array}$ & $\mathrm{d}_{90}(\mu \mathrm{m})$ & $\mathrm{d}_{50}(\mu \mathrm{m})$ & $\mathrm{d}_{10}(\mu \mathrm{m})$ & SPAN (-) & $\Phi(-)$ \\
\hline Açúcar cristal & $446,75(86,80)$ & 580 & 475 & 360 & 0,46 & 0,61 \\
Suglets ${ }^{\circledR}$ PF001 & $312,54(37,48)$ & 360 & 310 & 270 & 0,29 & 0,75 \\
Suglets ${ }^{\circledR}$ PF006 & $615,11(67,83)$ & 700 & 600 & 550 & 0,25 & 0,82 \\
Cel. microcristalina & $423,12(58,94)$ & 540 & 445 & 350 & 0,43 & 0,69 \\
Celphere ${ }^{\circledR}$ CP 305 & $422,40(47,36)$ & 460 & 430 & 380 & 0,19 & 0,86 \\
Celphere ${ }^{\circledR}$ CP 507 & $601,57(45,96)$ & 660 & 610 & 540 & 0,20 & 0,86 \\
Farinha mandioca & $466,80(74,45)$ & 555 & 480 & 385 & 0,35 & 0,66 \\
Arroz & $450,87(93,99)$ & 480 & 400 & 290 & 0,47 & 0,67 \\
\hline
\end{tabular}

$\mathrm{d}_{\mathrm{m}}=$ diâmetro médio das partículas; $\mathrm{d}_{90}=$ tamanho de partículas correspondente a $90 \%$ da distribuição de tamanho; $\mathrm{d}_{50}=$ tamanho de partículas correspondente a $50 \%$ da distribuição de tamanho; $\mathrm{d}_{10}=$ tamanho de partículas correspondente a $10 \%$ da distribuição de tamanho; SPAN = índice de polidispersidade; $\Phi=$ fator de forma.

As partículas de açúcar cristal e de arroz apresentaram maior polidispersidade, pois, devido às suas durezas, elas são quebradas em partes irregulares durante $\mathrm{o}$ processo de moagem, resultando em formas mais irregulares comparativamente à farinha de mandioca e de celulose microcristalina.

As partículas mais esféricas favorecem o processo de crescimento dos grânulos por revestimento (layering) por favorecer uma deposição mais homogênea da composição de alimentação sobre a superfície das partículas no leito fluidizado. As partículas mais irregulares tendem a favorecer o crescimento por aglomeração, formando grânulos maiores, que dependendo das condições operacionais utilizadas, podem levar à defluidização do sistema devido à formação de canais preferenciais de passagem do ar de fluidização, além da adesão de grandes aglomerados nas paredes da coluna devido à 
alta umidade no sistema. Em um estudo desenvolvido por Ramachandran et al. (2012), foi avaliado o efeito da polidispersão do tamanho de partículas sementes na homogeneidade dos grânulos formados, mostrando que a distribuição de tamanhos não teve efeito sobre a distribuição de tamanho dos grânulos, mas apresentou grande influência na composição e porosidade destes.
As partículas de açúcar, que apresentam estrutura cristalina e os pellets de açúcar e de celulose possuem maior densidade, seguidos pelo arroz, farinha de mandioca e celulose microcristalina, sendo que esta possui estrutura mais porosa e, portanto, menor densidade.

Tabela 2 - Caracterização físico-química das partículas inertes relacionadas à densidade e características de fluidez.

\section{Propriedades}

\begin{tabular}{ccccc}
\hline Partículas & $\rho b(\mathrm{~g} / \mathrm{mL})$ & $\rho \mathrm{T}, 1250(\mathrm{~g} / \mathrm{mL})$ & $\mathrm{FH}(-)$ & $\mathrm{IC}(\%)$ \\
\hline Açúcar cristal & 0,95 & 0,97 & 1,03 & 2,50 \\
Suglets ${ }^{\circledR}$ PF001 & 0,78 & 0,88 & 1,13 & 12,83 \\
Suglets ${ }^{\circledR}$ PF006 & 0,88 & 0,88 & 1,00 & 0,0 \\
Celulose microcristalina & 0,41 & 0,45 & 1,10 & 9,84 \\
Celphere ${ }^{\circledR}$ CP 305 & 0,93 & 0,99 & 1,10 & 6,45 \\
Celphere ${ }^{\circledR}$ CP 507 & 0,95 & 0,95 & 1,07 & 6,78 \\
Farinha mandioca & 0,60 & 0,66 & 1,10 & 10,34 \\
Arroz & 0,74 & 0,76 & 1,03 & 2,94 \\
\hline
\end{tabular}

$\rho b=$ densidade bruta; $\rho \mathrm{T}, 1250$ = densidade de compactação; $\mathrm{FH}=$ fator de Hausner; $\mathrm{IC}=$ índice de Carr.

A densidade de compactação relaciona-se com a perda por elutriação no sistema de fluidização, ou seja, quanto menor a densidade da partícula maior o arraste das partículas em relação às de densidade maior. Este fato interfere também na fluidodinâmica das partículas. Partículas menos densas, geralmente apresentam menor velocidade mínima de fluidização.

As propriedades de fluidez das partículas foram avaliadas baseadas no fator de Hausner e índice de Carr. Todas as partículas apresentaram fator de Hausner inferior a 1,25 e índice de Carr entre 0 e $16 \%$, indicando assim, excelente fluidez (Wells, 1988; De Jong, Hoffmann e Finkers, 1999).

A caracterização fluidodinâmica das diferentes partículas em leito fluidizado foi realizada para analisar o comportamento gássólido da fluidez das partículas. Esta análise é importante para determinação de parâmetros operacionais em um processo em leito fluidizado baseado nos valores experimentais da velocidade de mínima fluidização $\left(\mathrm{U}_{\mathrm{mf}}\right)$ obtidos através de intersecções da linha de leito fixo com a queda de pressão no leito $(\Delta \mathrm{P})$, procedimento proposto por Wen e $\mathrm{Yu}$ (1966). A velocidade mínima de fluidização é 
a velocidade necessária para que ocorra a suspensão inicial das partículas sementes, estabelecendo o limite inferior das velocidades operacionais, que para o processo de aglomeração/encapsulação em leito fluidizado é normalmente de cinco a seis vezes a velocidade mínima de fluidização (Parikh e Mogavero, 2005).

Os ensaios foram desenvolvidos adicionando-se $300 \mathrm{~g}$ e $600 \mathrm{~g}$ de cada partícula no interior do leito à temperatura ambiente $\left(25^{\circ} \mathrm{C}\right)$. A figura 1 apresenta um gráfico típico de queda de pressão no leito em função da velocidade de fluidização obtida para as partículas de Celphere ${ }^{\circledR}$ (CP-305 e CP-507). A tabela 3 apresenta as condições operacionais e os resultados experimentais da $\mathrm{U}_{\mathrm{mf}}$ para as diferentes partículas estudadas. Todos os resultados para as diferentes partículas foram semelhantes aos reportados na literatura para partículas classificadas como tipo B pelo diagrama simplificado de Geldard (Geldart, 1973; Olowson e Almstedt, 1991; Yates, 1998).

Tabela 3 - Condições operacionais e parâmetros fluidodinâmicos.

\begin{tabular}{ccccc}
\hline Partículas & $\mathrm{d}_{\mathrm{P}}(\mu \mathrm{m})$ & $\mathrm{M}_{0}(\mathrm{~g})$ & $\mathrm{H}_{0}(\mathrm{~cm})$ & $\mathrm{U}_{\mathrm{mf}}(\mathrm{m} / \mathrm{s})$ \\
\hline \multirow{2}{*}{ Açúcar cristal } & \multirow{2}{*}{$300-500$} & 300 & 4,0 & 0,05 \\
& & 600 & 7,3 & 0,06 \\
\hline \multirow{2}{*}{ Suglets ${ }^{\circledR}$ PF001 } & \multirow{2}{*}{$250-355$} & 300 & 4,3 & 0,03 \\
& & 600 & 8,0 & 0,04 \\
\hline \multirow{2}{*}{ Suglets ${ }^{\circledR}$ PF006 } & \multirow{2}{*}{$500-600$} & 300 & 4,2 & 0,07 \\
& & 600 & 8,0 & 0,08 \\
\hline \multirow{2}{*}{ Celulose microcristalina } & \multirow{2}{*}{$300-425$} & 300 & 6,5 & 0,03 \\
\hline \multirow{2}{*}{ Celphere ${ }^{\circledR} \mathrm{CP}-305$} & \multirow{2}{*}{$300-500$} & 300 & 4.0 & 0,04 \\
\hline \multirow{2}{*}{ Celphere ${ }^{\circledR} \mathrm{CP}-507$} & \multirow{2}{*}{$500-710$} & 300 & 4.5 & 0,06 \\
\hline \multirow{2}{*}{ Farinha de mandioca } & \multirow{2}{*}{$300-500$} & 600 & 7.5 & 0,06 \\
\hline \multirow{2}{*}{ Arroz } & \multirow{2}{*}{$300-500$} & 300 & 6,0 & 0,1 \\
\hline & & 600 & 9,3 & 0,03 \\
\hline
\end{tabular}

$\mathrm{d}_{\mathrm{p}}$ = faixa granulométrica; $\mathrm{M}_{0}=$ massa de partículas no leito; $\mathrm{H}_{0}=$ altura das partículas no leito; $\mathrm{U}_{\mathrm{mf}}=$ velocidade de mínima fluidização.

As partículas de maior granulometria e densidades, como os pellets de açúcar e de celulose, apresentaram maior velocidade mínima de fluidização.

Todas as partículas apresentaram bom comportamento fluidodinâmico, entretanto, as partículas de celulose microcristalina, que apresentaram baixa $\mathrm{U}_{\mathrm{mf}}$, baixa densidade e por serem mais porosas, tentem a sofrer maior perda por elutriação no processo em leito fluidizado, sendo necessárias alterações no sistema como a expansão ou aumento da altura da coluna, de modo a aumentar a área do leito diminuindo estas perdas. 
Os valores da $\mathrm{U}_{\mathrm{mf}}$ das partículas avaliadas também foram comparados com estimativas obtidas através de equações propostas por Wen e Yu (1966), Babu et al. (1978), Thonglimp (1981) e Lucas et al. (1986), derivadas da equação de Ergun. Essas equações estão apresentadas na Tabela 4.

$\mathrm{O}$ número de Arquimedes (Ar) e a $\mathrm{U}_{\mathrm{mf}}$ são definidos respectivamente como:

$$
A r=\frac{d_{P}^{3} \rho_{g}\left(\rho_{p}-\rho_{g}\right) g}{\mu^{2}}
$$

$$
U_{m f}=\frac{\mu \operatorname{Re}_{m f}}{d_{p} \rho_{g}}
$$

onde,

$\mathrm{d}_{\mathrm{P}}=$ diâmetro da partícula $(\mathrm{m})$;

$\rho_{\mathrm{g}}=$ densidade do fluido $\left(\mathrm{kg} \mathrm{m}^{-3}\right)$;

$\rho_{\mathrm{p}}=$ densidade da partícula $\left(\mathrm{kg} \mathrm{m}^{-3}\right)$;

$\mathrm{g}=$ acelaração gravitacional $\left(\mathrm{m} \mathrm{s}^{-1}\right)$;

$\mu=$ viscosidade do fluido ( $\mathrm{Pa} \mathrm{s}$ );

$\mathrm{Re}_{\mathrm{mf}}=$ número de Reynolds (-).

Os valores da $\mathrm{U}_{\mathrm{mf}}$ das partículas estimados através das equações da literatura e os valores experimentais obtidos neste estudo estão apresentados na Tabela 5.

Figura 1 - Resultados típicos da queda de pressão no leito em função da velocidade do gás de fluidização (pellets de celulose microcristalina de diferentes granulometrias).
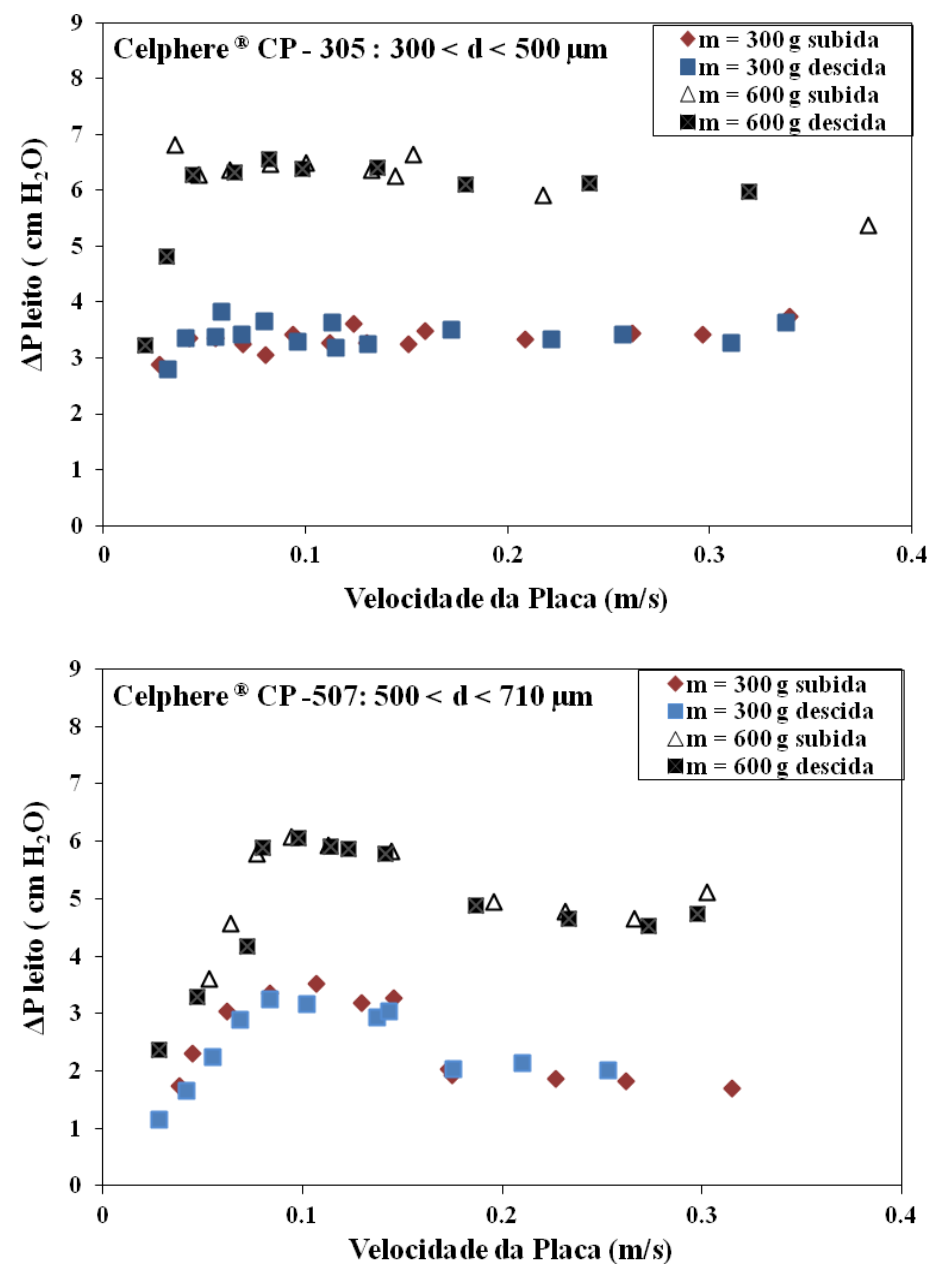
Tabela 4 - Equações empíricas para predição da $U_{\mathrm{mf}}$.

Autores

Wen e Yu, 1966

Babu et al., 1978

Thonglimp, 1981

Lucas et al., 1986

$$
\begin{gathered}
\text { Equações } \\
\operatorname{Re}_{\mathrm{mf}}=\left(33,7^{2}+0,0408 \mathrm{Ar}\right)^{1 / 2}-33,7 \\
\operatorname{Re}_{\mathrm{mf}}=\left(25,25^{2}+0,0651 \mathrm{Ar}\right)^{1 / 2}-25,25 \\
\operatorname{Re}_{\mathrm{mf}}=\left(31,6^{2}+0,0925 \mathrm{Ar}\right)^{1 / 2}-31,6 \\
\operatorname{Re}_{\mathrm{mf}}=\left(29,5^{2}+0,0357 \mathrm{Ar}\right)^{1 / 2}-29,5 \quad(0,8 \leq \Phi \leq 1) \\
\operatorname{Re}_{\mathrm{mf}}=\left(32,1^{2}+0,0571 \mathrm{Ar}\right)^{1 / 2}-32,1 \quad(0,5 \leq \Phi \leq 0,8) \\
\operatorname{Re}_{\mathrm{mf}}=\left(25,2^{2}+0,0672 \mathrm{Ar}\right)^{1 / 2}-25,2 \quad(0,1 \leq \Phi \leq 0,5)
\end{gathered}
$$

\section{Equações}

$\mathrm{Re}_{\mathrm{mf}}=$ número de Reynolds (adimensional); $\mathrm{Ar}=$ número de Arquimedes (adimensional); $\Phi=$ fator de forma.

Tabela 5 - Valores experimentais médios e estimados da $U_{\mathrm{mf}}$ das partículas analisadas.

$$
\mathrm{U}_{\mathrm{mf}}(\mathrm{m} / \mathrm{s})
$$

\begin{tabular}{cccccc}
\hline Partículas & $\begin{array}{c}\text { Valores experimentais } \\
\text { médios }\end{array}$ & $\begin{array}{c}\text { Wen e Yu } \\
(1966)\end{array}$ & $\begin{array}{c}\text { Babu } \text { et al. } \\
(1978)\end{array}$ & $\begin{array}{c}\text { Thonglimp, } \\
(1981)\end{array}$ & $\begin{array}{c}\text { Lucas } \text { et al. } \\
(1986)\end{array}$ \\
\hline Açúcar cristal & 0,06 & 0,06 & 0,11 & 0,14 & 0,08 \\
Suglets $^{\circledR}$ PF001 & 0,04 & 0,02 & 0,04 & 0,06 & 0,04 \\
Suglets $^{\circledR}$ PF006 & 0,08 & 0,10 & 0,19 & 0,22 & 0,10 \\
Celphere $^{\circledR} 305$ & 0,06 & 0,05 & 0,10 & 0,12 & 0,05 \\
Celphere $^{\circledR} 507$ & 0,10 & 0,10 & 0,20 & 0,23 & 0,10 \\
Cel. Microcrist. & 0,04 & 0,02 & 0,04 & 0,05 & 0,03 \\
Far. mandioca & 0,04 & 0,04 & 0,07 & 0,10 & 0,06 \\
Arroz & 0,07 & 0,05 & 0,09 & 0,11 & 0,07 \\
\hline
\end{tabular}

A maioria dos autores restringe o uso da equação de Ergun devido à dificuldade para determinar o fator de forma das partículas e a porosidade no leito, que fazem parte desta equação para determinar a $U_{\mathrm{mf}}$, usando então correlações experimentais obtidas com dados da literatura. Wen e Yu (1966) correlaciona dados para partículas com fator de forma igual a 1, ou seja, esfera perfeita, o que pode diminuir sua exatidão para partículas que apresentam fatores de forma inferiores (Lucas et al., 1986).
Lucas et al. (1986) visando a redução de erros na determinação da $\mathrm{U}_{\mathrm{mf}}$ para partículas classificadas de acordo com o fator de forma, usou a equação de Ergum como ponto inicial e a variação da porosidade do leito em função da forma da partícula estudada por Brownel et al. (1950), que apresentou concordância com dados experimentais determinados por Wen e Yu (1966). As partículas foram classificadas em três categorias de acordo com o fator de forma apresentado: partículas arredondadas, agudas e outras; e três equações foram obtidas 
através desta classificação para determinação da $U_{\mathrm{mf}}$, apresentadas na Tabela 4.

A comparação entre os valores experimentais e os valores estimados mostram que as correlações de Wen e Yu (1966) e Lucas et al. (1986) apresentaram melhor concordância com os desvios da ordem de 0 a $43 \%$ e de 0 a $71 \%$, respectivamente. Melhores concordâncias foram obtidas para as partículas de maiores fatores de forma que apresentaram menores desvios entre os valores experimentais e estimados.

As correlações obtidas com as equações de Babu et al. (1978) e Thonglimp (1981) apresentaram grandes desvios entre os valores experimentais e calculados, variando de 14 a $153 \%$ e 43 a $193 \%$, respectivamente. As diferenças observadas se devem a diferenças tanto das condições de processo, das características do sistema de fluidização e também devido às diferentes estruturas das partículas utilizadas, tornando assim, as correlações inadequadas.

\section{CONCLUSÕES}

Os resultados obtidos neste estudo demonstraram que as características físicoquímicas das partículas avaliadas são adequadas e apresentam boas características de fluidização, evidenciando potencial para serem utilizadas como partículas sementes em processos de aglomeração e/ou revestimento em leito fluidizado para obtenção de produtos com as características desejadas.

\section{REFERÊNCIAS}

AUlton, M. E. Pharmaceutics: The Science of Dosage Form Design. New York: Churchill \& Livingstone, 2002.
BABU, S. P.; SHAH, B.; TALWALKAR, A. AIChE Symposium Series, v. 74, n. 176, p. 176, 1978.

BENELLI, L. et al. Fluid bed drying and agglomeration of phytopharmaceutical compositions. Powder Technology, v. 273, p. 145-153, 2015.

BROWNELL, L. E.; DOMBROWSKI, H. S.; DICHEY, C. A. Chemical Engineering Progress, v. 46, n. 8, p. 415-422, 1950.

DE JONG, J. A. H.; HOFFMANN, A. C.; FINKERS, H. J. Properly determinie powder flowability to maximize plant output. Chemical Engineering Progress, v. 25, p. 34, 1999.

DIXIT, R.; PUTHLI, S. Fluidization Technologies: Aerodynamic Principles and Process Engineering. Journal of Pharmaceutical Sciences, v. 98, n. 11, p. 3933-3960, Nov 2009. ISSN 0022-3549. Disponível em: < <Go to ISI $>$ :/WOS:000271595800004 >.

GELDART, D. Types of Gas Fluidization. Powder Technology, v. 7, p. 285-292, 1973.

LUCAS, A. et al. Improved eqution for the calculation of minimum fluidization velocity. Industrial \& Engineering Chemistry Process Design and Development Journal, v. 25, n. 2, p. 426-429, 1986.

MURTHY, Z. V. P.; JOSHI, D. Fluidized bed drying of aonla (Emblica officinalis). Drying Technology, v. 25, n. 4-6, p. 883-889, 2007. ISSN 0737-3937. Disponível em: < <Go to ISI >://WOS:000247677200044 >.

MÖRL, L.; HEINRICH, S.; PEGLOW, M. Fluidized bed spray granulation. In: Salman, A. D.; Hounslow, M. J., et al. Handbook of Powder Technology, Granulation. First. 
Netherlands: Elsevier B. V., v.11, cap. 2, p.23-187, 2007.

NIAMNUY, C.; DEVAHASTIN, S. Drying kinetics and quality of coconut dried in a fluidized bed dryer. Journal of Food Engineering, v. 66, n. 2, p. 267-271, Jan 2005. ISSN 0260-8774. Disponível em: < $<$ Go to ISI >://WOS:000224283200017 >.

OLOWSON, P. A.; ALMSTEDT, A. E. Influence of pressure on the minimum fluidization velocity. Chemical Engineering Science, v. 46, n. 2, p. 637-640, 19911991. ISSN 0009-2509. Disponível em: < <Go to ISI >://WOS:A1991EU55800023 >.

PARIKH, D. M.; MOGAVERO, M. Batch Fluid Bed Granulation. In: Parikh, D. M. (Ed.). Handbook of pharmaceutical granulation technology. $2^{\text {th }}$ : Taylor \& Francis Group 2005. cap. 9, p.247-309.

RAMACHANDRAN, R. et al. A quantitative assessment of the influence of primary particle size polydispersity on granule inhomogeneity. Chemical Engineering Science, v. 71, p. 104-110, Mar 2012. ISSN 0009-2509. Disponível em: $<<$ Go to ISI $>: / / W O S: 000300235600012>$.

SALEH, K.; GUIGON, P. Coating and Encapsulation Process in Powder Techonology. In: Salman, A. D.;Hounslow, M. J., et al (Ed.). Granulatioin: Elsevier B. V., 2007. cap. 7, p.324-375.

THONGLIMP, V. Tese (Doutorado em Engenharia) - Institut National Polytechnique de Toulouse, France, 1981.

WELLS, J. I. Pharmaceutical Preformulation: The Physicochemical Properties of Drug Substances. New York: John Wiley \& Sons, 1988.
WEN, C. Y.; YU, Y. H. A generalized method for predicting the minimum fluidization velocity. AIChE Journal, v. 12, n. 3, p. 610-612, 1966.

YATES, J. G. Effects of temperature and pressure on gas-solid fluidization (vol 51, pg 167, 1996). Chemical Engineering Science, v. 53 , n. 10, p. 1955-1955, May 1998. ISSN 0009-2509. Disponível em: < <Go to ISI >://WOS:000074650900014 >.

YOSHINOBU, F.; HIDEKI, I. Fluid Bed Processes for Forming Functional Particles. In: Encyclopedia of Pharmaceutical Technology, Third Edition: Taylor \& Francis, p.1773-1778, 2013. ISBN 0-84939399-X.

ZILI, Z.; SOUAD, S.; FESSI, H. Preparation and characterization of poly- $\varepsilon$-caprolactone nanoparticles containing griseofulvin. International Journal of Pharmaceutics, v. 294, p. 261-267, 2005.

\section{AGRADECIMENTOS}

Os autores agradecem à FAPESP pelo apoio financeiro no desenvolvimento deste trabalho (Processo no 2012/00562-6). 